\title{
Eukaryotic translation initiation factor 3B downregulation inhibits cell proliferation and promotes cell apoptosis through negatively regulating tumor necrosis factor receptor superfamily member 21 in gastric cancer
}

\author{
Huiling Xiong ${ }^{1 \#}$, Mei Hu ${ }^{1 \#}$, Hui Huang ${ }^{2}$, Jing Gong ${ }^{1}$, Jie Wu ${ }^{1}$, Heng Zhang ${ }^{1}$ \\ ${ }^{1}$ Department of Gastroenterology, ${ }^{2}$ Department of Gastrointestinal Surgery, The Central Hospital of Wuhan, Tongji Medical College, Huazhong \\ University of Science and Technology, Wuhan 430014, China \\ Contributions: (I) Conception and design: H Zhang; (II) Administrative support: None; (III) Provision of study materials or patients: H Xiong, M Hu; \\ (IV) Collection and assembly of data: H Huang, J Gong; (V) Data analysis and interpretation: H Huang, J Wu; (VI) Manuscript writing: All authors; \\ (VII) Final approval of manuscript: All authors \\ \#These authors contributed equally to this work. \\ Correspondence to: Heng Zhang. Department of Gastroenterology, The Central Hospital of Wuhan, Tongji Medical College, Huazhong University of \\ Science and Technology, 26 Shengli Street, Wuhan 430014, China. Email: aojiang052047@163.com.
}

Background: This study aimed to detect eukaryotic translation initiation factor 3B (EIF3B) expression in gastric cancer (GC) cell lines, and further explore the effect of EIF3B downregulation on GC cell proliferation and apoptosis.

Methods: EIF3B mRNA expression and protein expression in human GC cell lines (NCI-N87, AGS, HGC-27, BGC-823 and MGC80-3) and human gastric mucosal epithelial cell line (GES-1) were detected. Control siRNA (Si-NC group) and EIF3B siRNA (Si-EIF3B group) were transfected into NCI-N87 cells. Rescue experiment was performed by transfection of EIF3B siRNA (Si-EIF3B group) and EIF3B siRNA plus tumor necrosis factor receptor superfamily member 21 (TNFRSF21) siRNA (Si-EIF3B \& Si-TNFRSF21 group) into NCI-N87 cells. Besides, cell proliferation, apoptosis, TNFRSF21 expression and TRAF1 expression were assessed.

Results: EIF3B mRNA expression and protein expression were elevated in NCI-N87, AGS, HGC-27 and BGC-823 cell lines compared to GES-1 cell line. In NCI-N87 cells, proliferation was reduced in Si-EIF3B group compared to Si-NC group. For cell apoptosis, its rate and apoptotic marker C-Caspase 3 expression were increased but anti-apoptosis marker Bcl-2 expression was reduced in Si-EIF3B group compared to SiNC group. Moreover, mRNA expression and protein expression of TNFRSF21 were increased in Si-EIF3B group compared to $\mathrm{Si}-\mathrm{NC}$ group, while mRNA expression and protein expression of TRAF1 were reduced in Si-EIF3B group compared to Si-NC group. In rescue experiment, cell proliferation was increased but apoptosis was decreased in Si-EIF3B \& Si-TNFRSF21 group compared to Si-EIF3B group.

Conclusions: EIF3B is overexpressed in GC cell lines, and its downregulation inhibits cell proliferation while promotes apoptosis through negatively regulating TNFRSF21 in GC.

Keywords: Cell apoptosis; cell proliferation; eukaryotic translation initiation factor 3B (EIF3B); gastric cancer (GC); tumor necrosis factor receptor superfamily member 21 (TNFRSF21)

Submitted Apr 28, 2019. Accepted for publication Aug 27, 2019.

doi: $10.21037 /$ tcr.2019.09.42

View this article at: http://dx.doi.org/10.21037/tcr.2019.09.42 


\section{Introduction}

Gastric cancer (GC), the fifth most common cancer and the third cause of cancer-related deaths, has been reported to cause approximately 1,000,000 new cases and estimated 783,000 deaths worldwide during $2018(1,2)$. Currently, dominant treatments for GC includes surgery, chemotherapy as well as radiotherapy, and there are great advances in these treatment methods in recent decades, whereas the 5 -year survival rate is just less than $20 \%$ and the prognosis of GC patients is still far from satisfactory $(2,3)$. Hence, it is urgently to investigate detailed mechanisms involved in the initiation and development of GC, which might contribute to exploring novel target for the GC treatment and improving the prognosis.

Eukaryotic translation initiation factors (EIFs) are well known to initiate protein translation by forming large complexes with ribosome and mRNA (4). As the largest member of EIFs, eukaryotic translation initiation factor 3 (EIF3) consists of 13 subunits and participates in almost all stages of initiation processes (5). Eukaryotic translation initiation factor 3B (EIF3B), an important scaffolding subunit among EIF3, is reported to be highly expressed in different cancers (such as esophageal squamous cell carcinoma and glioblastoma, colon cancer and bladder cancer), furthermore, EIF3B has shown oncogenic effect in various cancer cells (6-12). For instance, EIF3B knockdown represses cell proliferation and promotes cell apoptosis via activating caspase-3/poly ADP-ribose polymerase (PARP) pathway in osteosarcoma cells (10). Also, EIF3B knockdown has been reported to inhibit cell proliferation, enhance cell apoptosis and induce G0/G1 arrest through inhibiting DNA replication in glioma cells (6). Besides, a study discloses that EIF3B knockdown reduces cell proliferation, cell migration and cell invasion through inhibiting $\beta$-catenin pathway in renal cell carcinoma cells (12). Another interesting study shows that EIF3B enhances cell proliferation as well as cell invasion via facilitates activation of $\beta$-catenin signaling pathway in esophageal squamous cell carcinoma cells (7). Considering that EIF3B had carcinogenic effect in these cancers, we speculated that targeting EIF3B might have therapeutic function in GC, whereas related evidence was limited. Thus, we conducted this study to detect EIF3B expression in GC cell lines, and further explore the effect of EIF3B downregulation on GC cell proliferation and cell apoptosis.

\section{Methods}

\section{Cell lines}

Human GC cell lines NCI-N87, AGS, HGC-27, BGC-823, MGC80-3 and human gastric mucosal epithelial cell line GES-1 were purchased from Cell Bank of the Chinese Academy of Sciences (Shanghai, China). NCI-N87, HGC-27, BGC-823, MGC80-3 and GES-1 cells were cultured in 90\% Roswell Park Memorial Institute (RPMI) 1640 Medium (Gibco, USA) supplemented with $10 \%$ fetal bovine serum (Gibco, USA), while AGS cells were cultured in $90 \%$ Ham's F-12K (Kaighn's) Medium (Gibco, USA) supplemented with $10 \%$ fetal bovine serum (Gibco, USA). All cell lines were cultured in the incubators at $37^{\circ} \mathrm{C}$ under $95 \%$ air and $5 \% \mathrm{CO}_{2}$ condition. EIF3B mRNA expression in these cell lines was detected by quantitative polymerase chain reaction (qPCR), and its protein expression in each cell line was detected by Western Blot. The process of qPCR and Western Blot was shown in the followed subsections.

\section{EIF3B siRNA transfection and subsequent detections}

Control siRNA and EIF3B siRNA were established by Sangon Biotech (Shanghai) Co., Ltd (Shanghai, China). Then control siRNA and EIF3B siRNA were transfected into NCI-N87 cells by HilyMax (Dojindo, Japan) as SiNC group and Si-EIF3B group. Post transfection, EIF3B mRNA expression was detected at $24 \mathrm{~h}$ by qPCR, and EIF3B protein expression was detected at $24 \mathrm{~h}$ by Western Blot. Then cell proliferation ability was detected at 0, 24, 48 and 72 h using Cell Counting Kit -8 (Sangon, China) according to the instructions. Besides, cell apoptosis rate was detected at $24 \mathrm{~h}$ using Annexin V-FITC Apoptosis Detection Kit (R\&D, USA) according to the instructions. Finally, apoptosis markers [Cleaved Caspase 3 (C-Caspase 3) and B-cell lymphoma-2 (Bcl-2)] protein expressions were detected at $24 \mathrm{~h}$ by Western blot.

\section{Measurement of tumor necrosis factor receptor superfamily member 21 (TNFRSF21) and tumor necrosis factor receptor associated factor (TRAF1) expressions}

We analyzed the downstream genes and pathways of EIF3B using KEGG (https://www.kegg.jp/) and GeneCards (http:// www.genecards.org/), and we observed that EIF3B negatively 
Table 1 Primers list

\begin{tabular}{lll}
\hline Gene & Forward primer (5'-3') & Reverse primer $\left(5^{\prime}-3^{\prime}\right)$ \\
\hline EIF3B & CGTATGTGCGTTGGTCTCCTAA & CCTTGGTGGCTGAATCTCTGAA \\
TNFRSF21 & CCTTCTCCGCTGTGACTCTAC & CCTTCTCCGCTGTGACTCTAC \\
TRAF1 & CTGAATGGAGATGGCACTGGAA & TGCTCACGGTTGTTCTGGTC \\
GAPDH & GACCACAGTCCATGCCATCAC & ACGCCTGCTTCACCACCTT \\
\hline
\end{tabular}

EIF3B, eukaryotic translation initiation factor 3B; TNFRSF21, tumor necrosis factor receptor superfamily member 21; TRAF1, tumor necrosis factor receptor associated factor; GAPDH, glyceraldehyde-3-phosphate dehydrogenase.

Table 2 Antibodies list

\begin{tabular}{lll}
\hline Antibody & Company & Dilution \\
\hline Primary antibody & & \\
Rabbit monoclonal to elF3B & Abcam (UK) & $1: 10,000$ \\
Rabbit polyclonal to DR6 (TNFRSF21) & Abcam (UK) & $1: 1,000$ \\
Rabbit polyclonal to TRAF1 & Abcam (UK) & $1: 500$ \\
Rabbit monoclonal to Caspase-3 & CST (USA) & $1: 1,000$ \\
Rabbit monoclonal to Cleaved & CST (USA) & $1: 1,000$ \\
Caspase-3 & & \\
Rabbit monoclonal to Bcl-2 & CST (USA) & $1: 1,000$ \\
Rabbit monoclonal to GAPDH & CST (USA) & $1: 1,000$ \\
Secondary antibody & & \\
Goat anti-rabbit lgG H\&L (HRP) & CST (USA) & $1: 4,000$ \\
\hline
\end{tabular}

EIF3B, eukaryotic translation initiation factor 3B; TNFRSF21, tumor necrosis factor receptor superfamily member 21; TRAF1, tumor necrosis factor receptor associated factor; GAPDH, glyceraldehyde-3-phosphate dehydrogenase.

regulated TNFRSF21. And previous data revealed that EIF3B functions as an oncogene via negatively regulating TNFRSF21 in some cancers other than GC $(10,11)$. Therefore, post transfection of control siRNA and EIF3B siRNA into NCI-N87 cells, TNFRSF21 and its downstream gene TRAF1 expressions were detected at $24 \mathrm{~h}$ by qPCR and Western Blot.

\section{EIF3B siRNA plus TNFRSF21 siRNA transfection and subsequent detections}

EIF3B siRNA and TNFRSF21 siRNA were established by Sangon Biotech (Shanghai) Co., Ltd (Shanghai, China). Then EIF3B siRNA alone, and EIF3B siRNA plus TNFRSF2 1 siRNA were transfected into NCI-N87 cells by HilyMax (Dojindo, Japan) as Si-EIF3B group and Si-EIF3B \& Si-TNFRSF21 group. Post transfection,
mRNA expressions of EIF3B, TNFRSF21 and TRAF1 were detected at $24 \mathrm{~h}$, and protein expressions of EIF3B, TNFRSF 21 and TRAF1 were detected at $24 \mathrm{~h}$. Then cell proliferation ability was detected at $0,24,48$ and $72 \mathrm{~h}$. Besides, cell apoptosis rate was detected at $24 \mathrm{~h}$. Finally, apoptosis markers [Cleaved Caspase 3 (C-Caspase 3) and B-cell lymphoma-2 (Bcl-2)] protein expressions were detected at $24 \mathrm{~h}$. The detection methods and reagent kits used in this section was the same as "EIF3B siRNA transfection and subsequent detections" section.

\section{$q P C R$}

The qPCR assay was performed as following steps: firstly, total RNA was extracted by TRIzol ${ }^{\mathrm{TM}}$ Reagent (Invitrogen, USA); secondly, reverse transcription to cDNA was conducted using ReverTra Ace ${ }^{\circledR}$ qPCR RT Kit (Toyobo, Japan); thirdly, qPCR was performed using KOD SYBR ${ }^{\circledR}$ qPCR Mix (Toyobo, Japan). In addition, the $\mathrm{qPCR}$ results were calculated by $2^{-\Delta \Delta C t}$ formula, and glyceraldehyde-3-phosphate dehydrogenase (GAPDH) was applied as the internal references. Primers used in qPCR assay were listed in Table 1.

\section{Western blot}

Protein expression in each cell line was detected by Western Blot as following steps: firstly, total protein was extracted from cells using RIPA Lysis Buffer (Beyotime, China); secondly, total protein concentration was assessed using BCA Protein Assay Kit (Beyotime, China); thirdly, electrophoresis was performed on NuPAGE ${ }^{\mathrm{TM}} 4-12 \%$ BisTris Protein Gels (Invitrogen, USA), and then proteins were transferred to polyvinylidene fluoride (PVDF) membrane (Millipore, German); Fourthly, membranes were incubated with primary and secondary antibodies in turn. Finally, bands were visualized with X-ray film (Kodak, USA). Information of antibodies was listed in Table 2. 

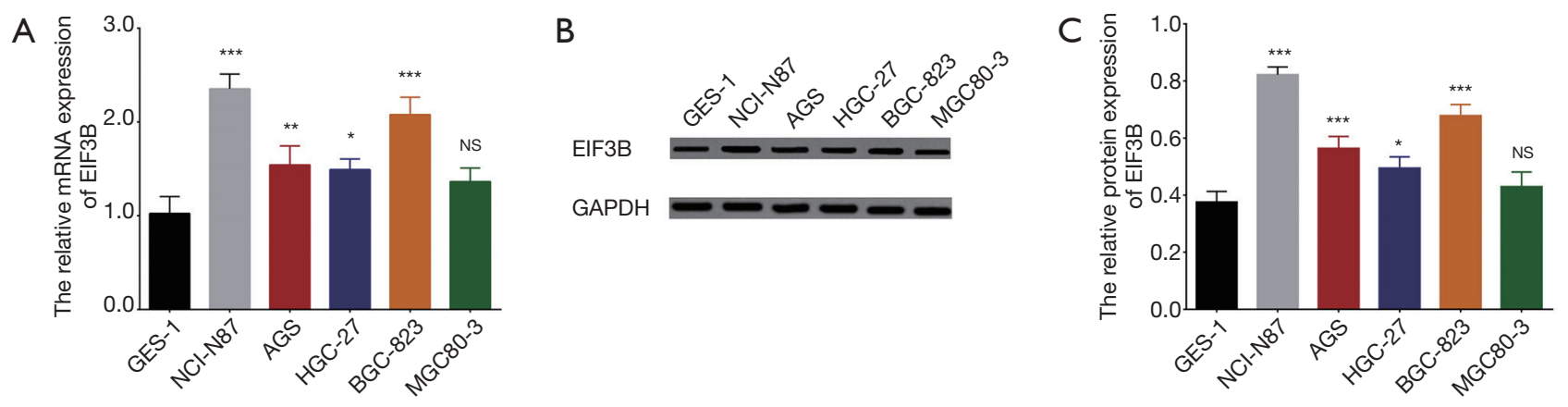

Figure 1 EIF3B in GC cell lines and control cell line. EIF3B mRNA expression in GC cell lines and control cell line (A). EIF3B protein expression in GC cell lines and control cell line (B,C). Comparison between two groups was determined by One-Way ANOVA test followed by multiple comparisons test (Dunnett- $t$-test). $\mathrm{P}$ value $<0.05$ was considered significant. ${ }^{* * *}, \mathrm{P}<0.001 ;{ }^{* *}, \mathrm{P}<0.01 ;{ }^{*}, \mathrm{P}<0.05$. NS, no significance; EIF3B, eukaryotic translation initiation factor 3B; GC, gastric cancer.

\section{Statistics}

Statistical analysis and graphs were performed using GraphPad Prism 7.01 Software (GraphPad Int., USA). Comparison among groups was detected by One-Way ANOVA test followed by multiple comparisons test (Dunnett- $t$-test), comparison between two groups was detected by $t$-test. $\mathrm{P}<0.05$ was considered as significant.

\section{Results}

Comparison of EIF3B expression between various human GC cell lines and human gastric mucosal epithelial cell line

EIF3B mRNA expression was elevated in various human GC cell lines including NCI-N87 $(\mathrm{P}<0.001)$, AGS $(\mathrm{P}<0.01)$, HGC-27 $(\mathrm{P}<0.05)$ and BGC-823 $(\mathrm{P}<0.001)$ compared to human gastric mucosal epithelial cell line GES-1 (Figure $1 A$ ), while no difference of EIF3B mRNA expression was found between MGC80-3 cell line compared to GES-1 cell line $(\mathrm{P}>0.05)$. Besides, protein expressions of EIF3B in NCI-N87, AGS, HGC-27 and BGC-823 cell lines were increased compared to GES-1 cells (all $\mathrm{P}$ values $<0.05$ ), while no difference of EIF3B protein expression was observed between MGC80-3 cell line and GES-1 cell line (Figure 1B,C). Considering the numerically highest EIF3B mRNA expression observed in NCI-N87 cells, we chose NCI-N87 cells to perform EIF3B downregulation related experiments.
Effect of EIF3B downregulation on cell proliferation and cell apoptosis in NCI-N87 cells

After EIF3B siRNA was transfected into NCI-N87 cells, mRNA $(\mathrm{P}<0.001)$ (Figure $2 A)$ and protein expressions $(\mathrm{P}<0.001)$ (Figure $2 B, C)$ of EIF3B were reduced in $\mathrm{Si}$ EIF3B group compared to Si-NC group. Moreover, cell proliferation was decreased in Si-EIF3B group compared to Si-NC group at $48 \mathrm{~h}(\mathrm{P}<0.05)$ and $72 \mathrm{~h}(\mathrm{P}<0.01)$ (Figure $2 D)$, while cell apoptosis rate was raised in Si-EIF3B group compared to Si-NC group at $24 \mathrm{~h}(\mathrm{P}<0.01)$ (Figure $2 E$ ), meanwhile, cell apoptotic marker $\mathrm{C}$-Caspase 3 expression $(\mathrm{P}<0.01)$ was increased but anti-apoptosis marker Bcl2 expression $(\mathrm{P}<0.001)$ was reduced in $\mathrm{Si}-\mathrm{EIF} 3 \mathrm{~B}$ group compared to Si-NC group at $24 \mathrm{~h}$ (Figure $2 F, G, H$ ), and flow cytometry analysis also showed the similar trends (Figure 2I). These data suggested that EIF3B downregulation repressed cell proliferation but enhanced cell apoptosis in NCI-N87 cells.

\section{Effect of EIF3B downregulation on expressions of TNFRSF21 and TRAF1 in NCI-N87 cells}

According to the published articles about EIF3B in cancers, we found that EIF3B negatively regulated TNFRSF21 in some cancers other than GC (10). To further explore the molecule mechanism of EIF3B downregulation in GC cells, we subsequently detected expressions of TNFRSF21 
A

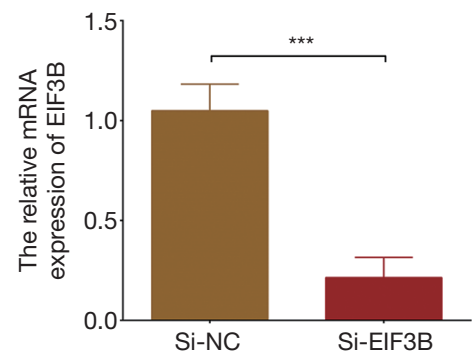

D

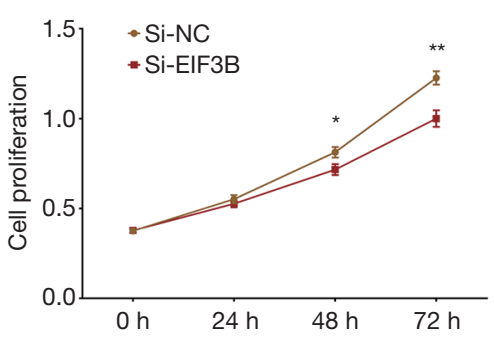

G

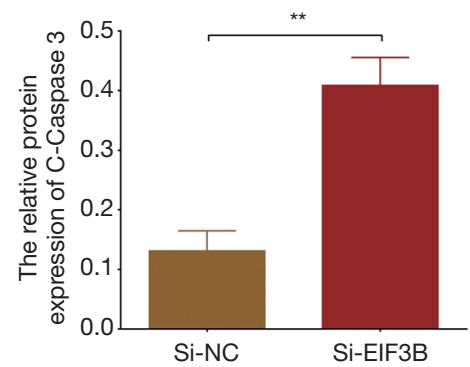

B

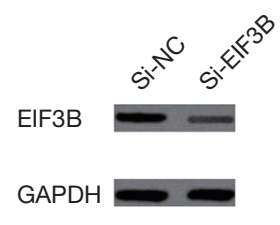

E

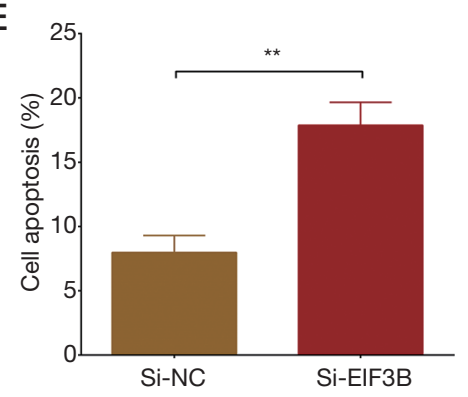

$\mathrm{H}$

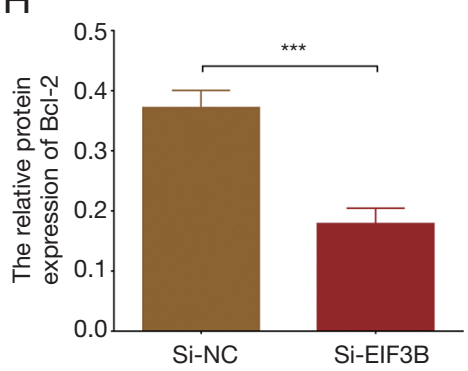

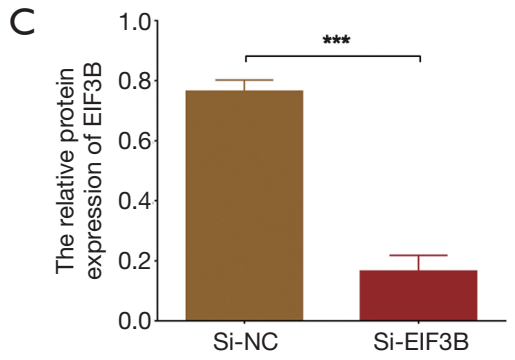

F

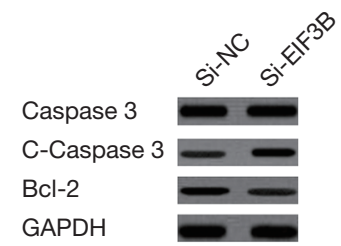

I

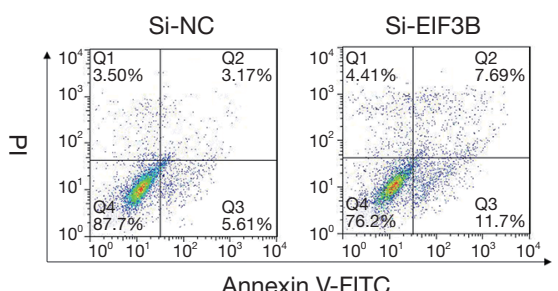

Figure 2 Cell proliferation and cell apoptosis in NCI-N87 cells by EIF3B downregulation. EIF3B mRNA expression was decreased in Si-EIF3B group compared to Si-NC group (A). EIF3B protein expression was lower in Si-EIF3B group compared to Si-NC group (B,C). Cell proliferation was reduced in Si-EIF3B group compared to Si-NC group (D). Cell apoptosis rate was higher in Si-EIF3B group compared to Si-NC group (E). Cell apoptosis-related marker expressions by Western blot assay (F,G,H). Flow cytometry analysis for cell apoptosis (I). P value $<0.05$ was considered significant. ${ }^{* * *}, \mathrm{P}<0.001$; ${ }^{* *}, \mathrm{P}<0.01 ;{ }^{*}, \mathrm{P}<0.05$. Comparison between two groups was determined by $t$-test. EIF3B, eukaryotic translation initiation factor 3B.

as well as its downstream gene TRAF1 in NCI-N87 cells. And we found that TNFRSF21 mRNA expression $(\mathrm{P}<0.01)$ (Figure $3 A$ ) was higher in Si-EIF3B group compared to SiNC group, while TRAF1 (which was negatively regulated by TNFRSF21) mRNA expression was reduced in SiEIF3B group compared to $\mathrm{Si}-\mathrm{NC}$ group $(\mathrm{P}<0.05)$ (Figure 3B). Meanwhile, the TNFRSF21 protein expression was elevated $(\mathrm{P}<0.001)$ but TRAF1 protein expression $(\mathrm{P}<0.05)$ was decreased in Si-EIF3B group compared to Si-NC group (Figure 3C,D,E). These data indicated that EIF3B downregulation upregulated TNFRSF21 expression in NCI-N87 cells.

\section{EIF3B affected GC cell functions through regulating TNFRSF 21}

In order to identify whether EIF3B affected cell functions of GC cell lines through regulating TNFRSF21, we performed rescue experiments in NCI-N87 cells: EIF3B siRNA alone, and EIF3B siRNA plus TNFRSF21 siRNA plasmids were transfected into NCI-N87 cells. As shown in Figure $4 A$, no difference of EIF3B mRNA expression was observed between Si-EIF3B \& Si-TNFRSF21 group and Si-EIF3B group $(\mathrm{P}>0.05)$. Whereas, compared to Si-EIF3B group, TNFRSF21 mRNA expression was decreased in Si- 
A

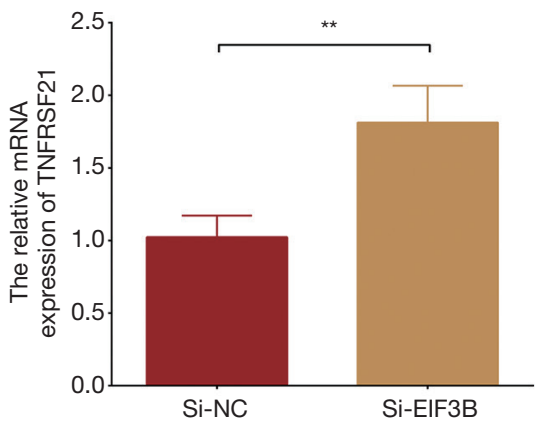

D

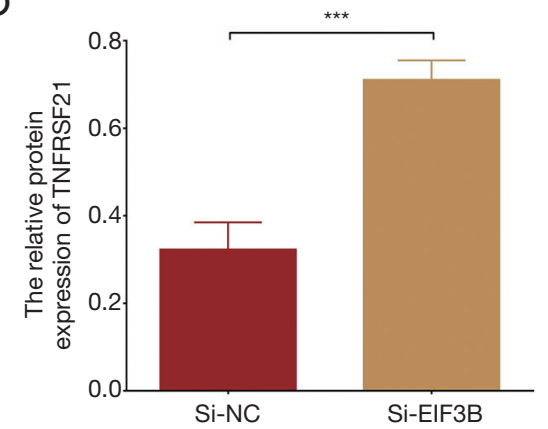

B

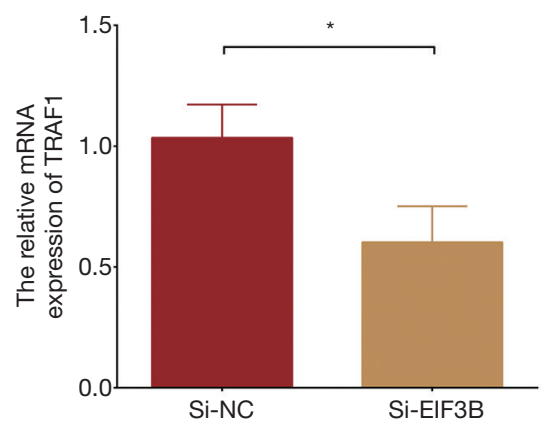

E

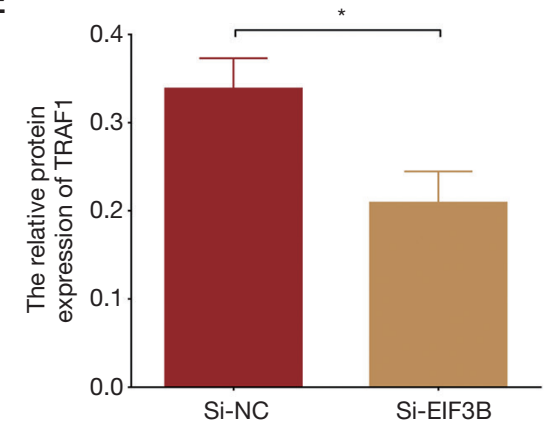

C

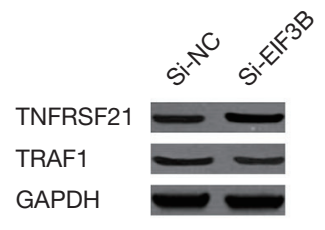

Figure 3 TNFRSF21 expression and its downstream gene expression in NCI-N87 cells by EIF3B downregulation. TNFRSF21 mRNA expression was increased in Si-EIF3B group compared to Si-NC group (A). TRAF1 mRNA expression was decreased in Si-EIF3B group compared to Si-NC group (B). TNFRSF21 protein expression was elevated but TRAF1 protein expression was reduced in Si-EIF3B group compared to Si-NC group (C,D,E). Comparison between two groups was determined by $t$-test. $\mathrm{P}$ value $<0.05$ was considered significant. ${ }^{* * *}$, $\mathrm{P}<0.001 ;{ }^{* *}, \mathrm{P}<0.01 ;{ }^{*}, \mathrm{P}<0.05$. TNFRSF21, tumor necrosis factor receptor superfamily member 21; EIF3B, eukaryotic translation initiation factor $3 \mathrm{~B}$; TRAF1, tumor necrosis factor receptor associated factor.

EIF3B \& Si-TNFRSF21 group $(\mathrm{P}<0.01)$ (Figure $4 B)$ but TRAF1 mRNA expression was enhanced in Si-EIF3B \& Si-TNFRSF21 group $(\mathrm{P}<0.05)$ (Figure $4 C)$. Meanwhile, the data of protein expressions showed the similar trend: no difference of EIF3B protein expression was found $(\mathrm{P}>0.05)$, but TNFRSF21 protein expression was decreased $(\mathrm{P}<0.01)$ in Si-EIF3B \& Si-TNFRSF21 group and TRAF1 protein expression was enhanced $(\mathrm{P}<0.001)$ in Si-EIF3B \& Si-TNFRSF21 group (Figure $4 D, E, F, G$ ). These results indicated that the transfection of aforementioned plasmids to NCI-N87 cells was successfully performed. In the subsequent assays, cell proliferation was enhanced in $\mathrm{Si}$ EIF3B \& Si-TNFRSF21 group compared to Si-EIF3B group at $48 \mathrm{~h}(\mathrm{P}<0.05)$ and $72 \mathrm{~h}(\mathrm{P}<0.05)$ (Figure $5 A)$, while cell apoptosis rate was decreased in Si-EIF3B \& Si-TNFRSF21 group compared to Si-EIF3B group at $24 \mathrm{~h}(\mathrm{P}<0.05)$ (Figure 5B). In addition, protein expression of cell apoptotic marker $\mathrm{C}$-Caspase 3 was reduced $(\mathrm{P}<0.001)$ but protein expression of anti-apoptosis marker Bcl-2 was increased $(\mathrm{P}<0.01)$ in Si-EIF3B \& Si-TNFRSF21 group compared to Si-EIF3B group (Figure 5C,D,E). Additionally, flow cytometry analysis also showed the decreased cell apoptosis in Si-EIF3B \& Si-TNFRSF21 group compared to Si-EIF3B group (Figure $5 F$ ). These data suggested that EIF3B downregulation might inhibit cell proliferation and promote cell apoptosis through negatively regulating TNFRSF21 in GC.

\section{Discussion}

In this study, we found that EIF3B was overexpressed in various human GC cell lines compared to human normal gastric epithelial cell line. Moreover, cell proliferation was decreased but cell apoptosis was promoted by EIF3B downregulation in NCI-N87 cells, and EIF3B downregulation might exert these functions through negatively regulating TNFRSF 21 .

EIFs, which are required in the synthesis of most 

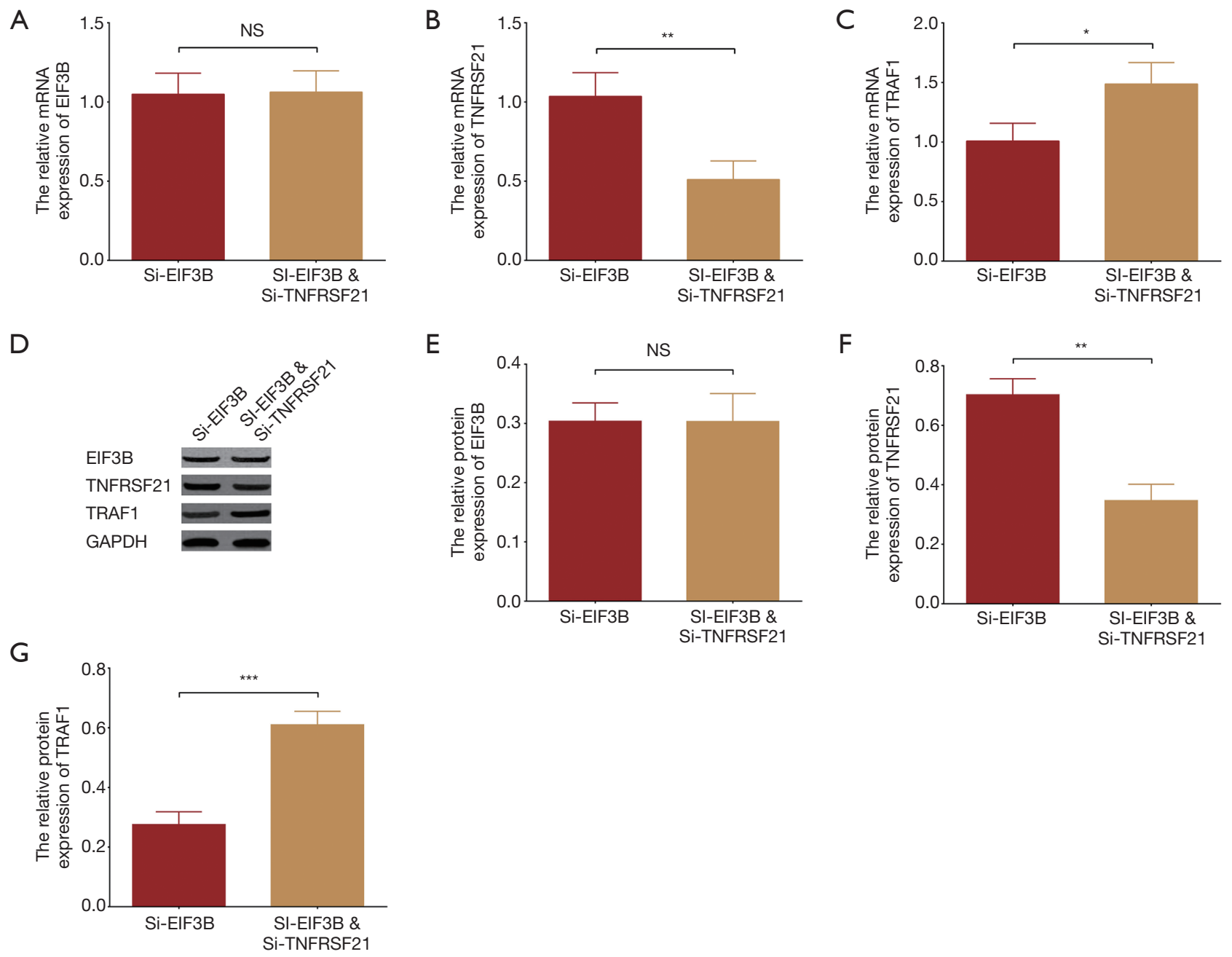

Figure 4 EIF3B, TNFRSF21 and TRAF1 expressions in NCI-N87 cells after EIF3B siRNA plus TNFRSF21 siRNA transfection. No difference of EIF3B mRNA expression was found between Si-EIF3B \& Si-TNFRSF21 group and Si-EIF3B group (A). TNFRSF21 mRNA expression was decreased in Si-EIF3B \& Si-TNFRSF21 group compared to Si-EIF3B group (B). TRAF1 mRNA expression was increased in Si-EIF3B \& Si-TNFRSF21 group compared to Si-EIF3B group (C). EIF3B protein expression was nondifferentiated, but TNFRSF21 protein expression was decreased and TRAF1 protein expressions were increased in Si-EIF3B \& Si-TNFRSF21 group compared to SiEIF3B group (D,E,F,G). Comparison between two groups was determined by $t$-test. $\mathrm{P}$ value $<0.05$ was considered significant. ***, $\mathrm{P}<0.001$; **, $\mathrm{P}<0.01$; * $\mathrm{P}<0.05$; NS, no significance; EIF3B, eukaryotic translation initiation factor $3 \mathrm{~B}$; TNFRSF21, tumor necrosis factor receptor superfamily member 21 ; TRAF1, tumor necrosis factor receptor associated factor.

proteins, have been reported to play a critical role in tumor initiation as well as progression, especially the EIF3 complex, which is the largest multi-subunit complex among EIFs $(12,13)$. For example, EIF3D knockdown inhibits cell proliferation as well as colony formation in vitro through upregulating stress-activated protein kinase (SAPK)/ c-Jun N-terminal kinase (JNK) pathway in colon cancer cells (5). Besides, EIF3D knockdown also suppresses breast cancer cell proliferation and invasion through inhibiting the $W n t / \beta$-catenin signaling pathway (14). For GC cells, $\mathrm{EIF} 3 \mathrm{H}$ knockdown represses cell proliferation as well as colony formation in GC cell lines but induces cell apoptosis through upregulating pro-apoptotic factor [Bcl-2-associated $\mathrm{X}$ protein $(\mathrm{BAX})]$ and downregulating anti-apoptotic factor [Bcl extra-large (Bcl-xL)] respectively, and causes cycle arrest at G0/G1 phase (4). These previous data reveal that 
A

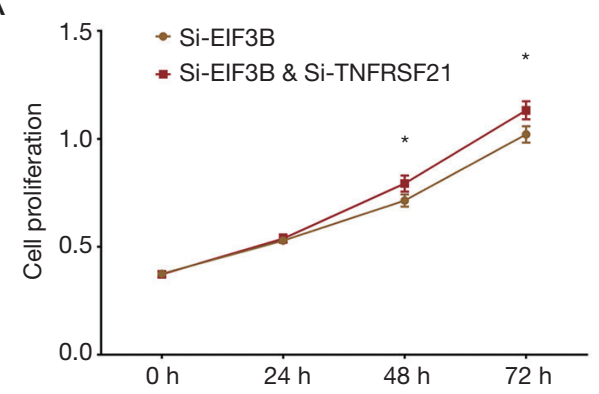

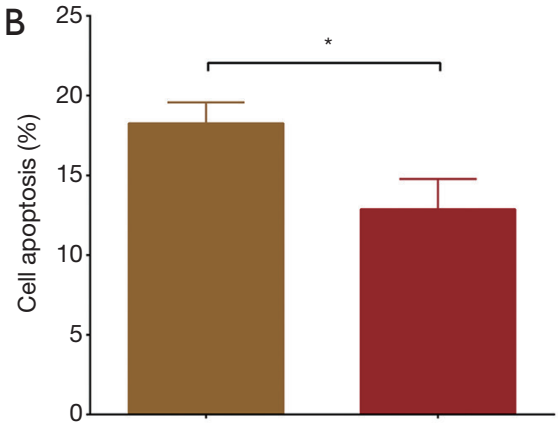

Si-EIF3B

SI-EIF3B \& Si-TNFRSF21

D

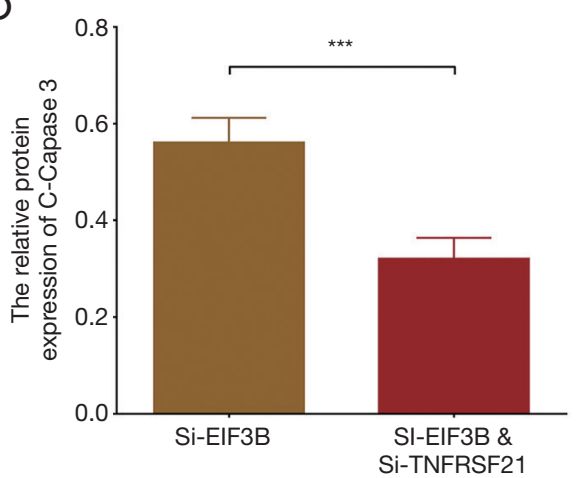

$\mathrm{F}$

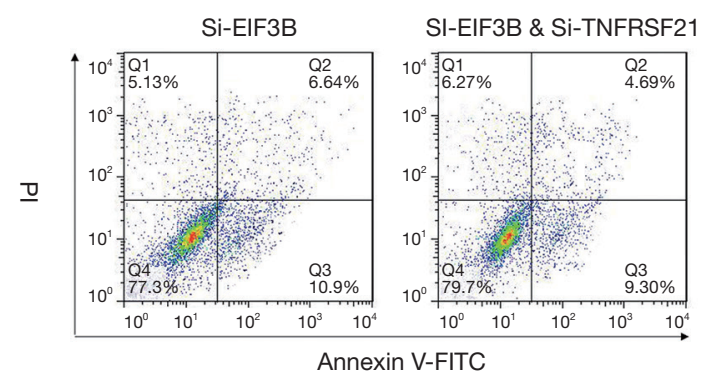

Figure 5 Cell proliferation and cell apoptosis in NCI-N87 cells after EIF3B siRNA plus TNFRSF21 siRNA transfection. Cell proliferation was increased in Si-EIF3B \& Si-TNFRSF21 group compared to Si-EIF3B group (A). Cell apoptosis was reduced in Si-EIF3B \& SiTNFRSF21 group compared to Si-EIF3B group at $24 \mathrm{~h}(\mathrm{P}<0.05)(\mathrm{B}, \mathrm{D})$. Cell apoptotic marker C-Caspase 3 was reduced but protein expression of anti-apoptosis marker Bcl-2 was increased in Si-EIF3B \& Si-TNFRSF21 group compared to Si-EIF3B group (C,D,E). Flow cytometry analysis for cell apoptosis $(\mathrm{F})$. Comparison between two groups was determined by $t$-test. $\mathrm{P}$ value $<0.05$ was considered significant. ${ }^{* *}, \mathrm{P}<0.001 ;{ }^{* *}, \mathrm{P}<0.01 ;{ }^{*}, \mathrm{P}<0.05$. EIF3B, eukaryotic translation initiation factor $3 \mathrm{~B}$; TNFRSF21, tumor necrosis factor receptor superfamily member 21 .

targeting EIFs may have therapeutic functions in these cancers.

EIF3B, a major scaffold protein of EIF3, has a particularly interesting characteristic that it presents a critical scaffolding function for the entire EIF3 complex
$(9,15)$. It is able to induce the formation of a $48 \mathrm{~S}$ preinitiation complex that could recognize AUG start codon, and it is considered to play a crucial role in both cellular and viral translation initiation (6). Moreover, recent studies disclose the regulatory effect of EIF3B in cancer 
cells $(6,7,10)$. For example, the inhibition of EIF3B on cell apoptosis through blocking caspase-3/PARP pathway in osteosarcoma cells, the enhancement of EIF3B on cell proliferation via activating $\beta$-catenin signaling pathway in esophageal squamous cell carcinoma cells, and the induction of EIF3B knockdown on cell G0/G1 arrest in glioma cells (6-10). All these data suggest that EIF3B has pro-proliferation or anti-apoptotic effect in these cancers. Considering EIF3B acts as an oncogene in the mechanisms of these cancers, we hypothesized that it might also play an oncogenic role in the pathology of GC cells, whereas related evidence was limited. In this study, we detected EIF3B expression in GC cells as well as normal gastric epithelial cells, and we found that EIF3B was overexpressed in various GC cell lines compared to normal gastric epithelial cell line. Most importantly, we investigated the regulatory effect of EIF3B downregulation on cell proliferation and apoptosis in GC cell line (NCI-N87 cells), and we found that EIF3B downregulation inhibited cell proliferation but enhanced cell apoptosis in NCI-N87 cells. The possible reasons for our results might be as follows: (I) EIF3B might promote cell proliferation and cell invasion through regulating $\beta$-catenin signaling pathway or caspase-3/PARP pathway, thereby facilitated the initiation of GC, thus EIF3B expression was elevated in GC cell lines compared to normal cells $(7,10)$; (II) downregulated EIF3B might fail to inhibiting DNA replication as well as inducing G0/G1 arrest, thus the cell apoptosis was promoted and cell proliferation was enhanced in GC cell lines (6). These data might shed a light on the treatment of GC due to the repression of EIF3B downregulation on cell proliferation and the promotion of EIF3B on cell apoptosis.

In order to further investigate the molecule mechanism of EIF3B knockdown in GC, we analyzed the downstream genes as well as pathways of EIF3B by KEGG (https:// www.kegg.jp/) and GeneCards (http://www.genecards.org/), and we found that EIF3B had a negative regulation on TNFRSF21. Moreover, based on previous studies that focus on EIF3B in cancers, we found EIF3B acts as an oncogene through negatively regulating TNFRSF21 in osteosarcoma cells (10). Since EIF3B downregulation repressed cell proliferation and promoted cell apoptosis in GC cells, and TNFRSF21 was negatively regulated by EIF3B according to previous data, we hypothesized that the EIF3B might affect GC cell functions through regulating TNFRSF21, while no current study disclosed the regulation of EIF3B on TNFRSF21 in GC cells. In this current study, we conducted rescue experiments to identify whether EIF3B affected GC cell functions through regulating TNFRSF21, and we observed that EIF3B downregulation suppressed cell proliferation but enhanced cell apoptosis via negatively regulating TNFRSF21 in NCI-N87 cells. As to the reason for these data, previous studies show reveal that TNFRSF2 1 possesses a death domain and is known as Death Receptor 6, which overexpression induces apoptosis in various cell types through activation of caspase- 3 , and these mechanisms might explain our results $(10,16-19)$. Our data might offer indications for further studies that investigate molecule mechanisms of EIF3B knockdown in GC cells and explore novel therapeutic target for GC.

\section{Conclusions}

In conclusion, EIF3B is overexpressed in GC cell lines, and its downregulation inhibits cell proliferation while promotes cell apoptosis through negatively regulating TNFRSF2 1 in GC, which sheds new light on the further application of EIF3B as a potentially novel treatment target for GC.

\section{Acknowledgments}

Funding: None.

\section{Footnote}

Conflicts of Interest: The authors have completed the ICMJE uniform disclosure form (available at http://dx.doi. org/10.21037/tcr.2019.09.42). The authors have no conflicts of interest to declare.

Ethical Statement: The authors are accountable for all aspects of the work in ensuring that questions related to the accuracy or integrity of any part of the work are appropriately investigated and resolved. The study was conducted in accordance with the Declaration of Helsinki (as revised in 2013). The institutional ethical approval and individual informed consent were waived due to the nature of the study.

Open Access Statement: This is an Open Access article distributed in accordance with the Creative Commons Attribution-NonCommercial-NoDerivs 4.0 International License (CC BY-NC-ND 4.0), which permits the noncommercial replication and distribution of the article with the strict proviso that no changes or edits are made and the original work is properly cited (including links to both the 
formal publication through the relevant DOI and the license). See: https://creativecommons.org/licenses/by-nc-nd/4.0/.

\section{References}

1. Bray F, Ferlay J, Soerjomataram I, et al. Global cancer statistics 2018: GLOBOCAN estimates of incidence and mortality worldwide for 36 cancers in 185 countries. CA Cancer J Clin 2018;68:394-424.

2. Torre LA, Bray F, Siegel RL, et al. Global cancer statistics, 2012. CA Cancer J Clin 2015;65:87-108.

3. Chen ZL, Qin L, Peng XB, et al. INHBA gene silencing inhibits gastric cancer cell migration and invasion by impeding activation of the TGF-beta signaling pathway. J Cell Physiol 2019;234:18065-74.

4. Wang $\mathrm{X}$, Wang $\mathrm{H}$, Zhao S, et al. Eukaryotic translation initiation factor EIF3H potentiates gastric carcinoma cell proliferation. Tissue Cell 2018;53:23-9.

5. Yu X, Zheng B, Chai R. Lentivirus-mediated knockdown of eukaryotic translation initiation factor 3 subunit D inhibits proliferation of HCT116 colon cancer cells. Biosci Rep 2014;34:e00161.

6. Liang H, Ding X, Zhou C, et al. Knockdown of eukaryotic translation initiation factors 3B (EIF3B) inhibits proliferation and promotes apoptosis in glioblastoma cells. Neurol Sci 2012;33:1057-62.

7. $\mathrm{Xu} F, \mathrm{Xu} \mathrm{CZ}, \mathrm{Gu}$ J, et al. Eukaryotic translation initiation factor $3 \mathrm{~B}$ accelerates the progression of esophageal squamous cell carcinoma by activating beta-catenin signaling pathway. Oncotarget 2016;7:43401-11.

8. Wang Z, Chen J, Sun J, et al. RNA interference-mediated silencing of eukaryotic translation initiation factor 3, subunit B (EIF3B) gene expression inhibits proliferation of colon cancer cells. World J Surg Oncol 2012;10:119.

9. Wang H, Ru Y, Sanchez-Carbayo M, et al. Translation initiation factor eIF3b expression in human cancer and its role in tumor growth and lung colonization. Clin Cancer Res 2013;19:2850-60.

Cite this article as: Xiong $\mathrm{H}, \mathrm{Hu} \mathrm{M}$, Huang $\mathrm{H}$, Gong J, $\mathrm{Wu}$ J, Zhang $\mathrm{H}$. Eukaryotic translation initiation factor 3B downregulation inhibits cell proliferation and promotes cell apoptosis through negatively regulating tumor necrosis factor receptor superfamily member 21 in gastric cancer. Transl Cancer Res 2019;8(6):2242-2251. doi: 10.21037/tcr.2019.09.42
10. Choi YJ, Lee YS, Lee HW, et al. Silencing of translation initiation factor eIF3b promotes apoptosis in osteosarcoma cells. Bone Joint Res 2017;6:186-93.

11. Mirzaei MR, Najafi A, Arababadi MK, et al. Altered expression of apoptotic genes in response to OCT4B1 suppression in human tumor cell lines. Tumour Biol 2014;35:9999-10009.

12. Zang Y, Zhang X, Yan L, et al. Eukaryotic Translation Initiation Factor $3 \mathrm{~b}$ is both a Promising Prognostic Biomarker and a Potential Therapeutic Target for Patients with Clear Cell Renal Cell Carcinoma. J Cancer 2017;8:3049-61.

13. Jackson RJ, Hellen CU, Pestova TV. The mechanism of eukaryotic translation initiation and principles of its regulation. Nat Rev Mol Cell Biol 2010;11:113-27.

14. Fan Y, Guo Y. Knockdown of eIF3D inhibits breast cancer cell proliferation and invasion through suppressing the Wnt/beta-catenin signaling pathway. Int J Clin Exp Pathol 2015;8:10420-7.

15. Herrmannova A, Daujotyte D, Yang JC, et al. Structural analysis of an eIF3 subcomplex reveals conserved interactions required for a stable and proper translation pre-initiation complex assembly. Nucleic Acids Res 2012;40:2294-311.

16. Zeng L, Li T, Xu DC, et al. Death receptor 6 induces apoptosis not through type I or type II pathways, but via a unique mitochondria-dependent pathway by interacting with Bax protein. J Biol Chem 2012;287:29125-33.

17. Mi S, Lee X, Hu Y, et al. Death receptor 6 negatively regulates oligodendrocyte survival, maturation and myelination. Nat Med 2011;17:816-21.

18. Pan G, Bauer JH, Haridas V, et al. Identification and functional characterization of DR6, a novel death domaincontaining TNF receptor. FEBS Lett 1998;431:351-6.

19. Nikolaev A, McLaughlin T, O'Leary DD, et al. APP binds DR6 to trigger axon pruning and neuron death via distinct caspases. Nature 2009;457:981-9. 